\title{
Production of transversely polarized $\Lambda$ hyperon from unpolarized quark fragmentation in the diquark model
}

\author{
Yongliang Yang ${ }^{* \dagger}$ \\ School of Physics, Southeast University, Nanjing 211189, China \\ E-mail: vangy Leseu.edu.cn
}

\section{Wenjuan Mao}

School of Physics and Telecommunication Engineering, Zhoukou Normal University, Zhoukou 466000, Henan, China

E-mail: wjmaodseu.edu.cn

\section{Zhun Lu}

School of Physics, Southeast University, Nanjing 211189, China

E-mail: zhunLudseu.edu.co

\section{Ivan Schmidt}

Departamento de Física, Universidad Técnica Federico Santa María, and Centro Científico-Tecnológico de Valparaíso, Casilla 110-V, Valparaíso, Chile

E-mail: ivan.schmidtausm.cl

\begin{abstract}
We study the spin-dependent (naive) T-odd fragmentation function $D_{1 T}^{\perp}$ in a spectator model to obtain the knowledge of the process for the transverse polarization of the $\Lambda^{0}$ hyperon produced fragmented from an unpolarized quark. We consider both scalar diquark and vector diquark to obtain the flavor content of $\Lambda$ fragmentation functions. We determine the values of the model parameters by fitting the unpolarized fragmentation function $D_{1}^{\Lambda}$ to the DSV parametrization for $D_{1}^{\Lambda}$. With the model results of these fragmentation functions, we also estimate the transverse polarizations $P_{T}^{\Lambda}$ in both semi-inclusive deep inelastic scattering and single inclusive $e^{+} e^{-}$annihilation.
\end{abstract}

XXVI International Workshop on Deep-Inelastic Scattering and Related Subjects (DIS2018)

16-20 April 2018

Kobe, Japan

\footnotetext{
*Speaker.

$\dagger$ This work is partially supported by the National Natural Science Foundation of China (NSFC) grant 11575043 , by the Fundamental Research Funds for the Central Universities of China, by Fondecyt (Chile) grants 1140390 and FB0821. Y. Yang is supported d by the Scientific Research Foundation of Graduate School of Southeast University (Grant No. YBJJ1770) and by the Postgraduate Research \& Practice Innovation Program of Jiangsu Province (Grant No. KYCX17_0043). W. Mao is supported by the NSFC (Grants No. 11605297 and No. 51702380), by the High-level Talents Research and Startup Foundation Projects for Doctors of Zhoukou Normal University (Grant No. ZKNUC2016014) and by the Fundamental Research Funds for the Central Universities of China.
} 


\section{Introduction}

The production of a polarized $\Lambda$ hyperon from unpolarized $p p$ collisions [ $[$, [ ] ] has become a long-standing challenge in high energy physics [[]]. In particular, a spin-dependent (naive) T-odd fragmentation function $D_{1 T}^{\perp}$ of the $\Lambda$ hyperon, can be used to describe the transverse polarization of the $\Lambda$ hyperon production. In this work, we perform the calculation of $D_{1 T}^{\perp}$ for light flavors using a spectator diquark model [四, 回]. In order to obtain the flavor content of $\Lambda$ fragmentation functions, in our calculation, both the scalar diquark and the vector diquark will be considered. With the same model and parameters, we also give the T-odd fragmentation function $D_{1 T}^{\perp}$ for up, down and strange quarks, by considering the gluon scattering effect. We also estimate the numerical results of the transverse polarization of the $\Lambda$ hyperon production both in the semi-inclusive deep inelastic scattering (SIDIS) $\ell p \rightarrow \ell^{\prime}+\Lambda^{\uparrow}+X$ and the single inclusive $e^{+} e^{-}$annihilation (SIA) $e^{+}, e^{-} \rightarrow \Lambda(\bar{\Lambda})+X$.

\section{Model calculation of unpolarized $\Lambda$ fragmentation functions}

In this section, we calculate the unpolarized TMD fragmentation function $D_{1}^{\Lambda}\left(z, k_{T}\right)$ in a spectator diquark model [四, 回], which includes both the spin 0 (scalar diquark) and spin 1 (axial-vector diquark) spectator systems [ []. The unpolarized TMD fragmentation function $D_{1}^{\Lambda}\left(z, k_{T}\right)$ can be obtained from the following trace [ [1], $\mathbf{\square}]$

$$
D_{1}^{\Lambda}\left(z, k_{T}\right)=\frac{1}{4} \operatorname{Tr}\left[\left(\Delta\left(z, k_{T} ; S_{\Lambda}\right)+\Delta\left(z, k_{T} ;-S_{\Lambda}\right)\right) \gamma^{-}\right] .
$$

Using spectator model, the expression of the $\Delta\left(z, k_{T} ; S_{\Lambda}\right)$ at tree level can be given as

$$
\Delta\left(z, k_{T} ; S_{\Lambda}\right)=\frac{g_{D}^{2}}{4(2 \pi)^{3}} \frac{\left(\not k+m_{q}\right)\left(\not P_{\Lambda}+M_{\Lambda}\right)\left(1+a_{D} \gamma_{5} \$_{\Lambda}\right)\left(\not k+m_{q}\right)}{(1-z) P_{\Lambda}^{-}\left(k^{2}-m_{q}^{2}\right)^{2}}
$$

with $k^{2}=\frac{z}{(1-z)} \boldsymbol{k}_{T}^{2}+\frac{m_{D}^{2}}{(1-z)}+\frac{M_{\Lambda}^{2}}{z}$ and $k^{-}=\frac{P_{\Lambda}^{-}}{z}$. The spin factor $a_{D}$ takes the values $a_{s}=1$ and $a_{v}=-\frac{1}{3}$, and $m_{q}, m_{D}$ and $M_{\Lambda}$ denote the masses of the parent quark, the spectator diquark and the fragmenting $\Lambda$ hyperon, respectively.

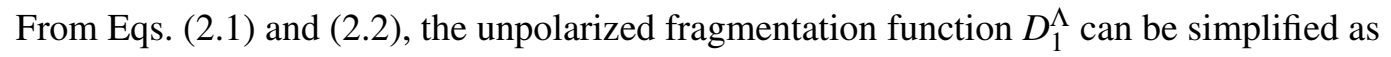

$$
D_{1}^{(s)}\left(z, z^{2} \boldsymbol{k}_{T}^{2}\right)=D_{1}^{(v)}\left(z, z^{2} \boldsymbol{k}_{T}^{2}\right)=\frac{g_{D}^{2}}{2(2 \pi)^{3}} \frac{(1-z)\left[z^{2} \boldsymbol{k}_{T}^{2}+\left(M_{\Lambda}+z m_{q}\right)^{2}\right]}{z^{4}\left(k_{T}^{2}+L^{2}\right)^{2}},
$$

with $L^{2}=\frac{1-z}{z^{2}} M_{\Lambda}^{2}+m_{q}^{2}+\frac{m_{D}^{2}-m_{q}^{2}}{z}$. In Eq. ([2.3),$D_{1}^{(s)}\left(z, z^{2} \boldsymbol{k}_{T}^{2}\right)$ and $D_{1}^{(v)}\left(z, z^{2} \boldsymbol{k}_{T}^{2}\right)$ denote the contributions to $D_{1}^{\Lambda}$ from the scalar diquark and the axial-vector diquark components, respectively. Under the summation for all polarization states of the axial-vector diquark: $\sum_{\lambda} \varepsilon_{\mu}^{*(\lambda)} \varepsilon_{v}^{(\lambda)}=-g_{\mu v}+\frac{P_{\Lambda \mu} P_{\Lambda v}}{M_{\Lambda}^{2}}$, the final results we get for them turn out to be the same.

In this work, we assume a SU(6) spin-flavor symmetry that the fragmentation functions of the $\Lambda$ hyperon for light flavors between the different quark flavors and diquark types satisfy the following relations $[[]]$ :

$$
D^{\mathrm{u} \rightarrow \Lambda}=D^{\mathrm{d} \rightarrow \Lambda}=\frac{1}{4} D^{(s)}+\frac{3}{4} D^{(v)}, D^{\mathrm{s} \rightarrow \Lambda}=D^{(s)},
$$


where $\mathrm{u}, \mathrm{d}$ and $\mathrm{s}$ denote the up, down and strange quarks, respectively. If the mass differences between the up, down and strange quarks are neglected, we can find that the light quarks fragment equals to $\Lambda$ for the unpolarized fragmentation function $D_{1}^{\Lambda}$, i.e.,

$$
D_{1}^{\mathrm{u} \rightarrow \Lambda}=D_{1}^{\mathrm{d} \rightarrow \Lambda}=D_{1}^{\mathrm{s} \rightarrow \Lambda} \equiv D_{1}^{\Lambda} .
$$

To obtain the integrated fragmentation function $D^{\Lambda}(z)$, we perform the integration over the transverse momentum of the produced hadron $\boldsymbol{P}_{T}=-z \boldsymbol{k}_{T}$ :

$$
D_{1}^{\Lambda}(z)=\int d^{2} \boldsymbol{P}_{T} D_{1}^{\Lambda}\left(z, \boldsymbol{P}_{T}^{2}\right)=\pi z^{2} \int_{0}^{\infty} d k_{T}^{2} D_{1}^{\Lambda}\left(z, z^{2} \boldsymbol{k}_{T}^{2}\right)
$$

here we choose a Gaussian form factor $g_{D} \mapsto \frac{g_{D}}{z} e^{-\frac{k^{2}}{\sqrt{2} \lambda^{2}}}$ to regularize the divergence from large values of $k_{T}$ when a point-like hyperon-quark-diquark coupling is considered. At last, the analytic result for $D_{1}^{\Lambda}(z)$ can be expressed as

$$
\begin{aligned}
D_{1}^{\Lambda}(z)= & \frac{g_{s}^{2}}{4(2 \pi)^{2}} \frac{e^{-\frac{2 m_{q}^{2}}{\Lambda^{2}}}}{z^{4} L^{2}}\left\{z(1-z)\left(\left(m_{q}+M_{\Lambda}\right)^{2}-m_{D}^{2}\right) \exp \left(\frac{-2 z L^{2}}{(1-z) \Lambda^{2}}\right)\right. \\
& \left.+\left((1-z) \Lambda^{2}-2\left(\left(m_{q}+M_{\Lambda}\right)^{2}-m_{D}^{2}\right)\right) \frac{z^{2} L^{2}}{\Lambda^{2}} \Gamma\left(0, \frac{2 z L^{2}}{(1-z) \Lambda^{2}}\right)\right\},
\end{aligned}
$$

where the incomplete gamma function has the form $\Gamma(0, z) \equiv \int_{z}^{\infty} \frac{e^{-t}}{t} d t$ and $\lambda, m_{D}, g_{s}$ are model parameters.

\section{Model calculation of the T-odd fragmentation function $D_{1 T}^{\perp}$}

In this section, we compute the T-odd TMD fragmentation function $D_{1 T}^{\perp}$ which describes the number density of a transversely polarized $\Lambda$ hyperon fragmented from an unpolarized quark [ $[$ ]. As is well known, T-odd fragmentation functions are vanishing in tree level calculation, its nonzero contribution comes from loop correction [ए]].

The TMD fragmentation function $D_{1 T}^{\perp}$ can be obtained from the following trace [W]:

$$
\frac{\varepsilon_{T}^{\rho \sigma} k_{T \rho} S_{\Lambda T \sigma}}{M_{\Lambda}} D_{1 T}^{\perp}\left(z, k_{T}\right)=\frac{1}{4} \operatorname{Tr}\left[\left(\Delta\left(z, k_{T} ; S_{\Lambda T}\right)-\Delta\left(z, k_{T} ;-S_{\Lambda T}\right)\right) \gamma^{-}\right] .
$$

Integrating over the loop momentum $l$ and using the Cutkosky cutting rules, we can first give the expression for $D_{1 T}^{\perp}$ from the scalar diquark component

$$
\begin{aligned}
D_{1 T}^{\perp(s)}\left(z, k_{T}^{2}\right) & =\frac{\alpha_{s} g_{s}^{2} C_{F}}{(2 \pi)^{4}} \frac{e^{\frac{-2 k^{2}}{\Lambda^{2}}}}{z^{2}(1-z)} \frac{1}{\left(k^{2}-m^{2}\right)} \\
& \times\left[D_{1 T(a)}^{\perp(s)}\left(z, k_{T}^{2}\right)+D_{1 T(b)}^{\perp(s)}\left(z, k_{T}^{2}\right)+D_{1 T(c)}^{\perp(s)}\left(z, k_{T}^{2}\right)+D_{1 T(d)}^{\perp(s)}\left(z, k_{T}^{2}\right)\right],
\end{aligned}
$$

where the four terms in the brackets correspond to the contributions from the four diagrams (plus their hermitian conjugates) at one loop level [ए2]:

$$
D_{1 T(a)}^{\perp(s)}\left(z, k_{T}^{2}\right)=\frac{m_{q} M_{\Lambda}}{\left(k^{2}-m_{q}^{2}\right)}\left(3-\frac{m_{q}^{2}}{k^{2}}\right) I_{1},
$$




$$
\begin{aligned}
D_{1 T(b)}^{\perp(s)}\left(z, k_{T}^{2}\right) & =M_{\Lambda}\left\{m_{q}\left(2 I_{2}-\mathscr{A}\right)-M_{\Lambda}\left(\mathscr{B}-2 I_{2}+2 \mathscr{A}\right)\right\}, \\
D_{1 T(c)}^{\perp(s)}\left(z, k_{T}^{2}\right) & =0, \\
D_{1 T(d)}^{\perp(s)}\left(z, k_{T}^{2}\right) & =\frac{M_{\Lambda}}{z}\left\{2(1-z)\left(m_{q} \mathscr{C} P_{\Lambda}^{-}-M_{\Lambda} \mathscr{D} P_{\Lambda}^{-}\right)-z\left(M_{\Lambda} \mathscr{B}-m_{q} \mathscr{A}\right)\right\},
\end{aligned}
$$

here $\mathscr{A}, \mathscr{B}, \mathscr{C}$ and $\mathscr{D}$ are functions of $k^{2}, m_{q}, m_{D}$ and $M_{\Lambda}$.

Similarly, we can give the expression for $D_{1 T}^{\perp}$ from the axial vector diquark component

$$
\begin{aligned}
D_{1 T}^{\perp(v)}\left(z, k_{T}^{2}\right) & =\frac{2 \alpha_{s} g_{s}^{2} C_{F}}{(2 \pi)^{4}} \frac{e^{\frac{-2 k^{2}}{\Lambda^{2}}}}{z^{2}(1-z)} \frac{1}{M_{\Lambda}\left(k^{2}-m_{q}^{2}\right)} \\
& \times\left[D_{1 T(v)}^{\perp(v)}\left(z, k_{T}^{2}\right)+D_{1 T(b)}^{\perp(v)}\left(z, k_{T}^{2}\right)+D_{1 T(c)}^{\perp(v)}\left(z, k_{T}^{2}\right)+D_{1 T(d)}^{\perp(v)}\left(z, k_{T}^{2}\right)\right],
\end{aligned}
$$

where the four terms in the r.h.s. of Eq. (B.]) can be written as

$$
\begin{aligned}
D_{1 T(a)}^{\perp(v)}\left(z, k_{T}^{2}\right) & =\frac{-m_{q} M_{\Lambda}}{(1-z)\left(k^{2}-m_{q}^{2}\right)}\left(1-\frac{m_{q}^{2}}{3 k^{2}}\right) I_{1}, \\
D_{1 T(b)}^{\perp(v)}\left(z, k_{T}^{2}\right) & =\frac{1}{3\left(k^{2}-m_{q}^{2}\right)}\left\{2 M_{\Lambda}\left[m_{q}\left(I_{2}-\mathscr{A}\right)+M_{\Lambda}\left(\mathscr{A}-I_{2}-\mathscr{B}\right)\right]+k \cdot P_{\Lambda}\left(4 I_{2}-6 \mathscr{A}\right)\right. \\
& \left.-\left(\mathscr{A} k \cdot P_{\Lambda}+\mathscr{B} P_{\Lambda}^{2}\right)+\frac{3}{2}\left(\frac{k^{2}-m_{q}^{2}}{2 k^{2}} I_{1}+\left(k^{2}-m_{q}^{2}\right) \mathscr{A}\right)\right\}, \\
D_{1 T(c)}^{\perp(v)}\left(z, k_{T}^{2}\right) & =0, \quad \\
D_{1 T(d)}^{\perp(v)}\left(z, k_{T}^{2}\right) & =\frac{-1}{3 M_{\Lambda}\left(k^{2}-m_{q}^{2}\right)}\left\{\left[M_{\Lambda}\left(\left(k^{2}-m_{q}^{2}\right) \mathscr{C} P_{\Lambda}^{-}+2 M_{\Lambda}^{2} \mathscr{D} P_{\Lambda}^{-}-2 m_{q} M_{\Lambda} \mathscr{C} P_{\Lambda}^{-}\right)\right.\right. \\
& \left.+2 k \cdot P_{\Lambda}\left(m_{q} \mathscr{C} P_{\Lambda}^{-}-M_{\Lambda} \mathscr{D} P_{\Lambda}^{-}\right)+z \frac{m_{q}}{2} I_{1}+\frac{\left(k^{2}-m_{q}^{2}\right)}{2}\left(M_{\Lambda} \mathscr{D} P_{\Lambda}^{-}-m_{q} \mathscr{C} P_{\Lambda}^{-}\right)\right] \\
& \left.-M_{\Lambda}\left(m_{q} M_{\Lambda} \mathscr{A}+2 k \cdot P_{\Lambda} \mathscr{A}+M_{\Lambda}^{2} \mathscr{B}\right)-\frac{2 M_{\Lambda}}{z}\left(m_{q} M_{\Lambda} \mathscr{C} P_{\Lambda}^{-}+k \cdot P_{\Lambda} \mathscr{C} P_{\Lambda}^{-}\right)\right\} .
\end{aligned}
$$

We assume that the relations in Eq. (ㄴ.4) also hold for the fragmentation function $D_{1 T}^{\perp}$ :

$$
D_{1 T}^{\perp \mathrm{u}}=D_{1 T}^{\perp d}=\frac{1}{4} D_{1 T}^{\perp(s)}+\frac{3}{4} D_{1 T}^{\perp(v)}, D_{1 T}^{\perp \mathrm{s}}=D_{1 T}^{\perp(s)},
$$

we can obtain $D_{1 T}^{\perp}$ for light flavors and calculate the half $k_{T}$-moment of $D_{1 T}^{\perp}$

$$
D_{1 T}^{\perp(1 / 2)}(z)=z^{2} \int d \boldsymbol{k}_{T}^{2} \frac{\left|\boldsymbol{k}_{T}\right|}{2 M_{\Lambda}} D_{1 T}^{\perp}\left(z, \boldsymbol{k}_{T}^{2}\right) .
$$

After the integration over $k_{T}^{2}$, one can get an approximate expression for the positivity bound in terms of $D_{1 T}^{\perp(1 / 2)}(z)$

$$
2 D_{1 T}^{\perp(1 / 2)}(z) \leq D_{1}(z)
$$

Using fitted results of the model parameters $g_{s}=1.983, m_{D}=0.745 \mathrm{GeV}$ and $\lambda=5.967 \mathrm{GeV}$, we give the numerical result of the half $k_{T}$-moment of the $\Lambda$ fragmentation function $D_{1 T}^{\perp}$ for light 

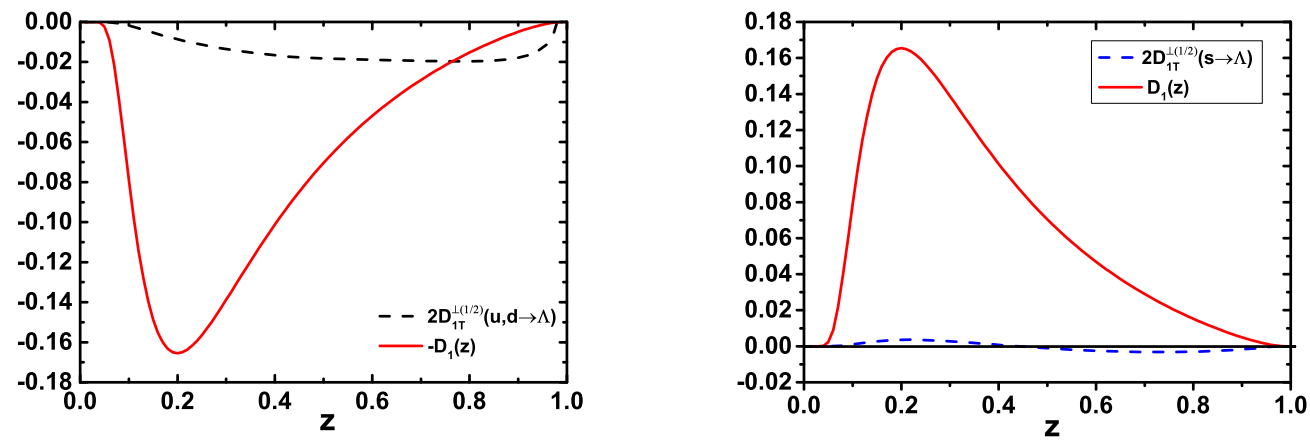

Figure 1: Left panel: the $D_{1 T}^{\perp(1 / 2)}(z)$ (multiplied by 2) (dashed line) and $D_{1}(z)$ (multiplied by -1) (solid line) of the up and down quark. Right panel: the $D_{1 T}^{\perp(1 / 2)}(z)$ (multiplied by 2) (dashed line) and $D_{1}(z)$ (solid line) of the strange quark.

flavors at the model scale $Q^{2}=0.23 \mathrm{GeV}^{2}$, by choosing the strong coupling constant at the model scale as $\alpha_{s}\left(\mu_{0}^{2}\right)=0.817$. We plotted the numerical results of $D_{1 T}^{\perp(1 / 2)}(z)$ (multiplied by a factor of 2) in Fig. $\mathrm{W}$. The unpolarized fragmentation function $D_{1}(z)$ (solid lines) is also plotted as the positivity bound for comparison.

Using the model result for $D_{1 T}^{\perp}$ of the $\Lambda$ hyperon, we predict the transverse $\Lambda$ polarization $P_{T}^{\Lambda}$ in SIDIS and SIA, which is a direct experimental observable. Generally, $P_{T}^{\Lambda}$ in high energy processes is defined as

$$
P_{T}^{\Lambda}=\frac{d \Delta \sigma}{d \sigma}=\frac{\left[d \sigma\left(S_{\Lambda T}\right)-d \sigma\left(-S_{\Lambda T}\right)\right]}{\left[d \sigma\left(S_{\Lambda T}\right)+d \sigma\left(-S_{\Lambda T}\right)\right]}
$$

Using the $S U(3)_{f}$ symmetric unpolarized $\Lambda$ fragmentation functions, i.e., $D_{1}^{u \rightarrow \Lambda} \equiv D_{1}^{d \rightarrow \Lambda} \equiv D_{1}^{s \rightarrow \Lambda}$, and ignoring the sea quarks $f_{\bar{q} / p}$ and strange quark $f_{s / p}$ contributions to SIDIS, we can obtain an approximate result for $P_{T}^{\Lambda}$ in SIDIS:

$$
\left.P_{T}^{\Lambda}\right|_{\mathrm{DIS}} \approx \frac{\Delta D_{\Lambda \uparrow / \mathrm{u}}}{D_{1}^{\mathrm{u} \rightarrow \Lambda}}
$$

Similarly, we can get a simplified form for $P_{T}^{\Lambda}$ in SIA:

$$
\left.P_{T}^{\Lambda}\right|_{\mathrm{SIA}}=\frac{5 \Delta D_{\Lambda^{\uparrow} / \mathrm{u}}+\Delta D_{\Lambda^{\uparrow} / \mathrm{s}}}{5 D_{1}^{\mathrm{u} \rightarrow \Lambda}+D_{1}^{\mathrm{s} \rightarrow \Lambda}}
$$

In Fig. $\downarrow$, we present the numerical result for the transverse $\Lambda$ polarization $P_{T}^{\Lambda}$ after averaging over the transverse momentum of the $\Lambda$ hyperon in both SIDIS (solid line) and SIA (dashed line). The results show that $P_{T}^{\Lambda}$ is negative and and substantial in the large $z$ region. Its size increases with increasing $z$ in both cases. Our results are consistent with the phenomenological analysis presented in Ref. [Q] and with the calculation of Ref. [13]. Furthermore, the shape of $P_{T}^{\Lambda}$ in SIA is very similar to that in SIDIS, as a consequence of the up and down quark dominance for $D_{1 T}^{\perp}$ in our model. As can be seen from Eqs. (B.T6) and (B.J7), the difference between $P_{T}^{\Lambda}$ in SIA and SIDIS is given by $D_{\Lambda^{\uparrow} / \mathrm{s}}$. We hope that the difference may provide a test for the strange quark contribution to $P_{T}^{\Lambda}$. 


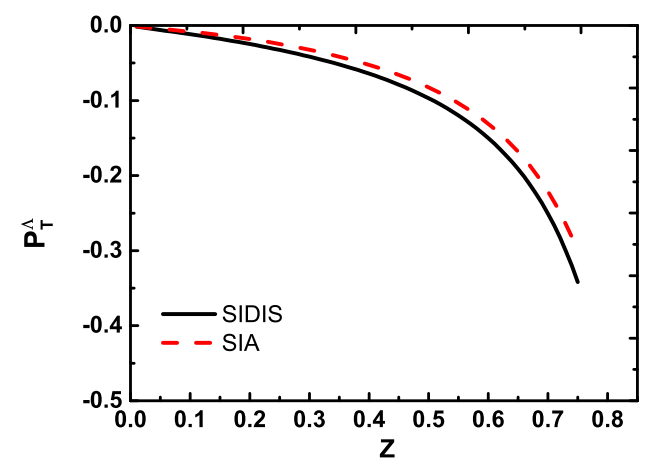

Figure 2: The transverse $\Lambda$ polarizations, $P_{T}^{\Lambda}$ vs $z$, averaged over $P_{\Lambda T}$, for SIDIS (solid line) and SIA (dashed line).

\section{Discussion and Conclusion}

In this work, we computed the T-odd fragmentation function $D_{1 T}^{\perp}$ using the diquark spectator model and studied its contribution to the transversely polarized $\Lambda$ production in SIDIS and SIA. We included in the calculation both the scalar diquark and the axial-vector diquark. We also applied the relation between the quark flavors and diquark types for fragmentation functions, motivated by the SU(6) symmetric wave functions of the $\Lambda$ hyperon. The values of the model parameters were determined by fitting the model result of unpolarized fragmentation function $D_{1}(z)$ with the DSV parametrization for $D_{1}$ at the initial scale $\mu_{0}^{2}=0.23 \mathrm{GeV}^{2}$. Using our model result of $D_{1 T}^{\perp}$, we estimated the transverse polarization $P_{T}^{\Lambda}$ in SIDIS and SIA, and found the polarizations are negative and substantial in the large $z$ region.

\section{References}

[1] A. Lesnik et al., Phys. Rev. Lett. 35, 770 (1975).

[2] G. Bunce et al., Phys. Rev. Lett. 36, 1113 (1976).

[3] W. G. D. Dharmaratna and G. R. Goldstein, Phys. Rev. D 53, 1073 (1996).

[4] M. Nzar and P. Hoodbhoy, Phys. Rev. D 51, 32 (1995).

[5] R. Jakob, P. J. Mulders and J. Rodrigues, Nucl. Phys. A 626, 937 (1997).

[6] A. Bacchetta, F. Conti and M. Radici, Phys. Rev. D 78, 074010 (2008).

[7] V. Barone, A. Drago and P. G. Ratcliffe, Phys. Rept. 359, 1 (2002).

[8] D. S. Hwang, arXiv:1608.02734.

[9] M. Anselmino, D. Boer, U. D’Alesio and F. Murgia, Phys. Rev. D 65, 114014 (2002).

[10] S. J. Brodsky, D. S. Hwang and I. Schmidt, Phys. Lett. B 530, 99 (2002)

[11] M. Anselmino, D. Boer, U. D’Alesio and F. Murgia, Phys. Rev. D 63, 054029 (2001).

[12] Y. Yang, Z. Lu and I. Schmidt, Phys. Rev. D 96, no. 3, 034010 (2017).

[13] D. Boer, Z. B. Kang, W. Vogelsang and F. Yuan, Phys. Rev. Lett. 105, 202001 (2010). 